\title{
Title: In vivo Chemical Reprogramming of Astrocytes into Functional
}

\section{Neurons}

Authors: Yantao $\mathrm{Ma}^{1,3, *}$, Handan Xie ${ }^{1, *}$, Xiaomin $\mathrm{Du}^{1,4,6, *}$, Lipeng Wang ${ }^{1, *}$, Xueqin Jin ${ }^{5, *}$, Shicheng Sun, Yanchuang Han, Yawen Han ${ }^{1,3}$, Jun $\mathrm{Xu}^{1}$, Zhuo Huang ${ }^{5, \ddagger}$, Zhen Chai ${ }^{6, \ddagger}$ and Hongkui Deng ${ }^{1,2, \neq}$

\begin{abstract}
Affiliations:
${ }^{1}$ Department of Cell Biology, School of Basic Medical Sciences, Peking University Stem Cell Research Center, State Key Laboratory of Natural and Biomimetic Drugs, Peking University Health Science Center, Beijing 100191, China; MOE Key Laboratory of Cell Proliferation and Differentiation, College of Life Sciences, Peking-Tsinghua Center for Life Sciences, Peking University, Beijing 100871, China.

${ }^{2}$ Shenzhen Stem Cell Engineering Laboratory, Key Laboratory of Chemical Genomics, Peking University Shenzhen Graduate School, Shenzhen, Guangdong 518055, China.
\end{abstract}

${ }^{3}$ Academy for Advanced Interdisciplinary Studies, Peking University, Beijing 100871, China. Peking University-Tsinghua University-National Institute of Biological Science Joint Gradute Program, College of Life Science, Peking University, Bejing 100871, China.

${ }^{4}$ Peking University-Tsinghua University-National Institute of Biological Science Joint Gradute Program, College of Life Science, Peking University, Bejing 100871, China.

${ }^{5}$ Department of Molecular and Celluar Pharmacology, School of Pharmaceutical Sciences, Health Science Center of Peking University, Bejing 100871, China.

${ }^{6}$ State Key Laboratory of Membrane Biology, College of Life Sciences, Peking University, Beijing 100871, China. 
bioRxiv preprint doi: https://doi.org/10.1101/305185; this version posted April 20, 2018. The copyright holder for this preprint (which was not certified by peer review) is the author/funder. All rights reserved. No reuse allowed without permission.

*These authors contributed equally to this work.

‡Corresponding author. Email: hongkui_deng@pku.edu.cn (H.D.); zhenchai@pku.edu.cn (Z.C.); huangz@hsc.pku.edu.cn (Z.H.) 
Abstract: Mammals lack robust regenerative abilities. Lost cells in impaired tissue could potentially be compensated by converting nearby cells in situ through in vivo reprogramming. Small molecule-induced reprogramming is a spatiotemporally flexible and non-integrative strategy for altering cell fate, which is, in principle, favorable for the in vivo reprogramming in organs with poor regenerative abilities, such as the brain. Here, we demonstrate that in the adult mouse brain, small molecules can reprogram resident astrocytes into functional neurons. The in situ chemically induced neurons (CiNs) resemble endogenous neurons in terms of neuron-specific marker expression and electrophysiological properties. Importantly, these CiNs can integrate into the mouse brain. Our study, for the first time, demonstrates in vivo chemical reprogramming in the adult brain, which could be a novel path for generating desired cells in situ for regenerative medicine.

\section{Main Text:}

The fate of committed cells can be reprogrammed by cellular, genetic and chemical approaches, which offers the possibility of generating desired cells by cell fate conversion (1-5). Remarkably, advances in chemical approaches have revealed that somatic cells can be reprogrammed into pluripotent stem cells completely by small molecules (5), and importantly, chemical reprograming is capable of inducing direct lineage conversion between differentiated cell types (6-10). Small molecules are easily manipulated and flexibly combined to reprogram cell identities at both the temporal and spatial levels to generate functional cells $(11,12)$. Furthermore, chemical approaches are cell permeable, reversible and non-integrative to the genome, which is favorable for in vivo reprogramming. Chemically induced in vivo reprogramming could, in principle, produce functional cells by converting nearby cells in situ to compensate for the cellular loss in damaged organs with limited regenerative capability, such as those of the central nervous systems (CNS). The mammalian brain does not readily repair damage because the loss of non-proliferative neurons is irreversible, and neural stem cells can only give rise to neurons in few brain regions. However, upon brain injury, neighboring astrocytes can become proliferative and serve as an abundant resource for reprogramming (13). For these reasons, our study aims to chemically reprogram local astrocytes in the adult brain, which could potentially regenerate compensatory neurons in situ. 


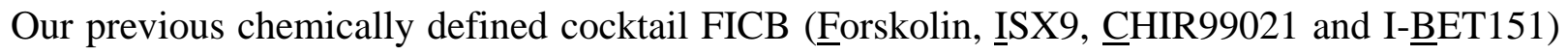
could reprogram fibroblasts into neuron-like cells in vitro (7). We therefore tested this cocktail on astrocytes carrying neuron-specific TauEGFP reporter for neuronal induction (14) (fig. S1). Upon exposure to FICB, astrocytes underwent neuron-like morphological changes with neurite outreach, and TauEGFP ${ }^{+}$cells were observed at 16 day-post-induction (dpi), suggesting the acquisition of neuronal cell fate. An optimized cocktail DFICBY (dbcAMP, Forskolin, ISX9, CHIR99021, I-BEET151 and $\underline{Y}$-27632) enhanced neuronal conversion efficiency, yielding 89.2 $\pm 1.4 \%$ of $\mathrm{TUJ}^{+}$cells with a neuron-like morphology at $16 \mathrm{dpi}$ (fig. S1, B and C), which cells were immunopositive for the neuron-specific markers MAP2, SYN1, and NF-H (fig. S1B). The mature neuronal marker NEUN was detected at 30 dpi with an efficiency of $77.8 \pm 11.1 \%$, when these cells were co-cultured with primary astrocytes from 16 dpi in a defined maturation medium

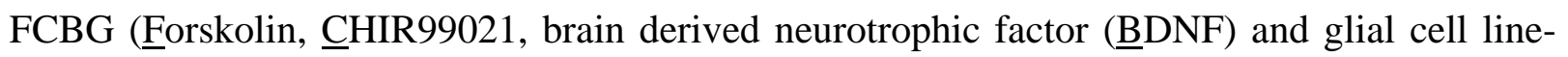
derived neurotrophic factor ( $\underline{G} D N F)$ ) for an additional two weeks. Meanwhile, the glutamatergic neuronal marker vGLUT2 and GABAergic neuronal marker GAD67 were also detected, suggesting that both excitatory and inhibitory neurons were generated (fig. S1, B and E). Wholecell recording showed that the CiNs were capable of generating action potentials (APs) in response to depolarizing step currents (fig. S1F, $\mathrm{n}=12$ ) and inactivating inward currents (fig. $\mathrm{S} 1 \mathrm{~F}$; Table $\mathrm{S} 1 ; \mathrm{n}=11$ ). We also confirmed the astroglial origin of CiNs with lineage-tracing mice carrying mGfap-Cre/Rosa26-tdTomato/TauEGFP $(15,16)$ (fig. S2, A and B). Nearly all $(96.5 \pm 1.1 \%)$ tdTOMATO ${ }^{+}$cells were immunopositive for astroglia-specific marker (S100B) and negative for neural markers (NEUN, DCX), microglial marker (IBA1) and oligodendrocyte marker (O4) (fig. S2, B and C). At 16 dpi with DFICBY treatment, we observed that $97.4 \pm 1.2$ $\%$ of TUJ1 ${ }^{+}$cells co-expressed tdTOMATO, suggesting that nearly all CiNs were converted from astrocytes (fig. S2D). Collectively, DFICBY, that robustly reprogrammed astrocytes into CiNs with functional neuronal properties.

Next, we tested whether the chemical cocktail could also induce CiNs from astrocytes in situ in adult mouse brains. To trace the astroglial origin of CiNs in vivo, we generated lineage-tracing mice by crossing mGfap-Cre mice with Rosa26-tdTomato reporter mice (Fig. 1A). In the crossed mice, tdTOMATO ${ }^{+}$cells in the striatum co-expressed the astrocyte markers GFAP $(95.6 \pm 4.0$ $\%)$ and S100B (91.3 $\pm 7.0 \%)$ and were negative for microglia marker IBA1 and 
oligodendrocyte marker MBP, suggesting a high specificity of the lineage-tracing system (Fig. 1, B and C). Rare tdTOMATO ${ }^{+}$cells (0.8 $\left.\pm 0.2 \%\right)$ expressed the neuronal cell marker NEUN but not proneural markers DCX, NEUROD1 and NEUROG2 (Fig. 1, B and C). Thus, initiating astrocytes for the in vivo reprogramming in the striatum of adult mouse brain were specifically labeled by tdTOMATO in mGfap-Cre/Rosa26-tdTomato mice.

To induce chemical reprogramming in vivo, the small molecule cocktail was administered into the striatum of 8-week old mice at a constant rate for two weeks via an osmotic mini-pumping system (Fig. 2A). After DFICBY induction, we observed tdTOMATO ${ }^{+} / \mathrm{NEUN}^{+}$cells in the striatum at 8 week-post-injection (wpi), suggesting that this cocktail could reprogram astrocytes into neuron-like cells in vivo (data not shown). We further optimized the DFICBY cocktail for in vivo reprogramming and found that partial substitution of dbcAMP for FSK did not overcome FSK to the efficiency of in vivo conversion. Additionally, we found that increasing the dose of FICBY by up to threefold in the in vitro dose led to a higher efficacy in vivo (fig. S3A). We next analyzed the effect of individual small molecules on the in vivo neuronal reprogramming. By single small molecule omission, we found that removing Forskolin, I-BET or ISX9 greatly hindered the generation of CiNs in vivo; and in the absence of CHIR99021, we observed a significant reduction in reprogramming efficiency (fig. S3B), suggesting that small molecules reprogrammed astrocytes to CiNs in vivo in a synergetic manner. At 8 wpi, the optimized formula FICBY generated tdTOMATO ${ }^{+} / \mathrm{NEUN}^{+}$cells in the striatum (Fig. 2B).

During this reprogramming process, at 2 wpi, the tdTOMATO ${ }^{+}$cells were immunopositive for the immature neuronal maker DCX and showed a mixed astrocyte-to-neuron morphology (Fig. 2C). At 5 wpi, tdTOMATO ${ }^{+} / \mathrm{NEUN}^{+}$cells were detected. The number of $\mathrm{NEUN}^{+} \mathrm{CiNs}$ increased from 5 to 8 wpi, suggesting gradual maturation of the CiNs after chemical induction (Fig. 2D). At 8 wpi, the tdTOMATO ${ }^{+}$cells also expressed other neuronal-specific markers including TUJ1 and NF-H (Fig. 2C). Interestingly, tdTOMATO ${ }^{+}$cells co-expressing the neuronal subtype marker GAD67 were also detected, suggesting that the small molecules could induce inhibitory CiNs from astrocytes in vivo (Fig. 2C).

To systemically quantify CiNs distribution, we analyzed 5 brain slices per mouse throughout the 
rostral-caudal extent on either side of the cannula at 8 wpi, which was considered the main distribution region of the small molecules (Fig. 2E). Scattered CiNs were distributed locally throughout the outer layer of the injection site, suggesting a restrained effective area of small molecules in the brain (Fig. 2B). Notably, $127 \pm 24$ tdTOMATO $^{+} / \mathrm{NEUN}^{+}$cells per slice $(\mathrm{n}=5)$ were observed at the injection core, while only a few tdTOMATO ${ }^{+} / \mathrm{NEUN}^{+}$cells were observed in the vehicle group ( $3 \pm 1$ tdTOMATO $^{+} / \mathrm{NEUN}^{+}$cells, $\mathrm{n}=5$ ) and intact group $(2 \pm 1$ tdTOMATO ${ }^{+} / \mathrm{NEUN}^{+}$cells, $\mathrm{n}=3$ ) (Fig. 2E). $\mathrm{NEUN}^{+} / \mathrm{tdTOMATO}^{+}$cell number did not show significant difference between intact group and vehicle group, which indicates surgery operation did not induce astrocyte-to-neuron cell fate conversion in situ (Fig. 2E). Furthermore, we observed tdTOMATO ${ }^{+} / \mathrm{NEUN}^{+} \mathrm{CiNs}$ one year-post-injection, suggesting its long-term survival capacity (Fig. 2C). Notably, no solid brain tumors were observed in any of the tested mice.

Next, we tested whether the in situ-generated CiNs displayed electrophysiological functions. We performed dynamic analyses of brain slice recordings on tdTOMATO ${ }^{+}$cells in the striatum with neuronal morphology around the injection site (Fig. 3A). Initially, at 0 wpi we randomly patched astrocytes on brain slices of reporter mice and found not competent for generating AP (Fig. 3C). Interestingly at $4 \mathrm{wpi}$, we observed membrane depolarization subjected to input current but still unable to generate AP, suggesting a transient state during the conversion toward neuronal electrophysiological property (Fig. 3B). AP of CiNs appeared as early as 6 wpi, followed by continuous APs at 8 wpi with a resting membrane potential of $-74.5 \pm 2.6 \mathrm{mV}$, an amplitude of $102.2 \pm 3.5 \mathrm{mV}$ and a threshold of $-41.7 \pm 5.3 \mathrm{mV}$ (Fig. 3C; Table S2; $\mathrm{n}=14$ ). The percentage of CiNs which could fire more than two spikes increased along time (Fig. 3D). From 4 to 12 wpi, we observed the gradual maturation of membrane-intrinsic properties, as indicated by increased membrane capacitance $(\mathrm{Cm})$ and a decrease in input resistance and AP threshold, which suggested that the CiNs resembled endogenous neurons over time (Fig. 3E; Table S2, 3). In addition, fast, inactivating inward currents were recorded in the CiNs at $8 \mathrm{wpi}$, which corresponds with the opening of voltage-dependent $\mathrm{Na}^{+}$channels (Fig. 3F; $\mathrm{n}=3$ ). We further detected spontaneous excitatory post-synaptic currents (EPSCs) in the CiNs by blocking the inhibitory inputs, which was subsequently blocked by 6-cyano-7-nitroquinoxaline-2,3-dione (CNQX) and 2-amino-5phospho-novaleric acid (AP5), resulting in the complete disappearance of EPSCs (Fig. 3G; n = 3). 
Collectively, these results indicated that in the mouse brain, CiNs acquired electrophysiological functions and formed synaptic connections.

Because the integration of compensatory neurons into the host neural circuitry is crucial for functional rescue (17), we further examined the connectivity of CiNs by a retrograde transsynaptic tracing system (18). We used a pseudotyped rabies virus (PRV) that specifically infected neurons expressing avian tumor virus receptor A (TVA). Subsequently, PRV assembled into infectious particles to cross one synapse where the rabies virus glycoprotein (RVG) was present. To achieve this, two Cre-dependent FLEX-AAV vectors encoding TVA, histone-EGFP and RVG were injected into the cortex of mGfap-Cre mice, resulting in their expressions restricted in astrocytes (Fig. 4A and fig. S4-5). After chemical induction, we injected PRV-carrying DsRed (PRV-DsRed) at 7 wpi into the same site to label the CiNs as DsRed ${ }^{+} / \mathrm{EGFP}^{+}$cells and to trace endogenous host neurons that made synaptic contact with CiNs as DsRed single-positive cells (Fig. 4A). Besides, we found that after TVA injection, cortical astrocytes were specifically labeled while with minor specificity within the corpus callosum of the striatum. Therefore, we did transsynaptic experiments in the cortex.

At 8 wpi, we observed DsRed ${ }^{+} / \mathrm{EGFP}^{+} \mathrm{CiNs}$ (22 \pm 2 cells in total) at the injection site in the cortex of the chemical induction group (Fig. 4, B and C). Meanwhile, DsRed single-positive cells were detected in the area surrounding the injection site (Fig. 4, B and D). The presence of both DsRed $^{+} / \mathrm{EGFP}^{+}$and DsRed single-positive cells in the chemical treated group indicated that the CiNs were able to be innervated by host neurons (Fig. 4, E and F). In contrast, there were no DsRed $^{+} /$EGFP $^{+}$cells observed in the vehicle group (Fig. 4C, $\mathrm{n}=4$ ). We also observed direct neurite connections between DsRed ${ }^{+} / \mathrm{EGFP}^{+} \mathrm{CiNs}$ and DsRed single-positive cells, suggesting that synapses formed between CiNs and host neurons (Fig. 4, E and F). These data suggest that in situ converted CiNs could timely receive synaptic signals from the local neurons in the adult brain.

Our study is the first to demonstrate that defined combinations of small molecules can induce the in vivo chemical reprogramming of astrocytes into functional mature neurons with electrophysiological characteristics. Importantly, these in situ-generated CiNs could functionally 
interact with resident neurons in the brain. Astrocytes serve as a latent plastic cell type for neurogenesis (19-21), and our work suggests a new strategy of in situ chemical reprogramming for the endogenous repair of brain injuries and neurodegenerative diseases. Future studies with disease models are needed to investigate the neuronal subtype-specific functions of these CiNs and to develop safety chemical delivery systems to different regions of the CNS. In vivo chemical reprogramming avoids the shortcomings of cell transplantation, including immunorejection, stem cell mutagenesis, inadequate cell survival and native tissue integration difficulties $(22,23)$. Moreover, small molecule-induced in situ lineage conversion is non-immunogenic and transgene free, which is eventually preferable for in vivo therapeutic strategies(24-28). Our finding may also offer a general strategy for in vivo chemical reprogramming to produce cells of other lineages. In vivo chemical reprogramming would open a novel path for regenerative medicine.

\section{References and Notes:}

1. J. B. Gurdon, T. R. Elsdale, M. Fischberg, Sexually mature individuals of Xenopus laevis from the transplantation of single somatic nuclei. Nature 182, $64-65$ (1958).

2. C. A. Cowan, J. Atienza, D. A. Melton, K. Eggan, Nuclear reprogramming of somatic cells after fusion with human embryonic stem cells. Science 309, 1369-1373 (2005).

3. K. Takahashi, S. Yamanaka, Induction of pluripotent stem cells from mouse embryonic and adult fibroblast cultures by defined factors. Cell 126, 663-676 (2006).

4. J. Yu et al., Induced pluripotent stem cell lines derived from human somatic cells. Science 318, 1917-1920 (2007).

5. P. Hou et al., Pluripotent stem cells induced from mouse somatic cells by small-molecule compounds. Science 341, 651-654 (2013).

6. W. Hu et al., Direct Conversion of Normal and Alzheimer's Disease Human Fibroblasts into Neuronal Cells by Small Molecules. Cell Stem Cell 17, 204-212 (2015).

7. X. Li et al., Small-Molecule-Driven Direct Reprogramming of Mouse Fibroblasts into Functional Neurons. Cell Stem Cell 17, 195-203 (2015).

8. L. Zhang et al., Small Molecules Efficiently Reprogram Human Astroglial Cells into Functional Neurons. Cell Stem Cell, (2015).

9. N. Cao et al., Conversion of human fibroblasts into functional cardiomyocytes by small 
molecules. Science 352, 1216-1220 (2016).

10. X. Li et al., Direct Reprogramming of Fibroblasts via a Chemically Induced XEN-like State. Cell Stem Cell 21, 264-273.e267 (2017).

11. W. Li, K. Li, W. Wei, S. Ding, Chemical approaches to stem cell biology and therapeutics. Cell Stem Cell 13, 270-283 (2013).

12. J. Xu, Y. Du, H. Deng, Direct lineage reprogramming: strategies, mechanisms, and applications. Cell Stem Cell 16, 119-134 (2015).

13. M. V. Sofroniew, H. V. Vinters, Astrocytes: biology and pathology. Acta neuropathologica 119, 7-35 (2010).

14. K. L. Tucker, M. Meyer, Y. A. Barde, Neurotrophins are required for nerve growth during development. Nat Neurosci 4, 29-37 (2001).

15. Y. Lee, A. Messing, M. Su, M. Brenner, GFAP promoter elements required for regionspecific and astrocyte-specific expression. Glia 56, 481-493 (2008).

16. L. Madisen et al., A robust and high-throughput Cre reporting and characterization system for the whole mouse brain. Nat Neurosci 13, 133-140 (2010).

17. S. Falkner et al., Transplanted embryonic neurons integrate into adult neocortical circuits. Nature 539, 248-253 (2016).

18. I. R. Wickersham et al., Monosynaptic restriction of transsynaptic tracing from single, genetically targeted neurons. Neuron 53, 639-647 (2007).

19. J. P. Magnusson et al., A latent neurogenic program in astrocytes regulated by Notch signaling in the mouse. Science 346, 237-241 (2014).

20. M. Gotz, S. Sirko, J. Beckers, M. Irmler, Reactive astrocytes as neural stem or progenitor cells: In vivo lineage, In vitro potential, and Genome-wide expression analysis. Glia 63, 1452-1468 (2015).

21. G. Nato et al., Striatal astrocytes produce neuroblasts in an excitotoxic model of Huntington's disease. Development 142, 840-845 (2015).

22. V. Tabar, L. Studer, Pluripotent stem cells in regenerative medicine: challenges and recent progress. Nat Rev Genet 15, 82-92 (2014).

23. J. Mertens, M. C. Marchetto, C. Bardy, F. H. Gage, Evaluating cell reprogramming, differentiation and conversion technologies in neuroscience. Nat Rev Neurosci 17, 424437 (2016). 
24. Q. Zhou, J. Brown, A. Kanarek, J. Rajagopal, D. A. Melton, In vivo reprogramming of adult pancreatic exocrine cells to beta-cells. Nature 455, 627-632 (2008).

25. L. Qian et al., In vivo reprogramming of murine cardiac fibroblasts into induced cardiomyocytes. Nature 485, 593-598 (2012).

26. O. Torper et al., Generation of induced neurons via direct conversion in vivo. Proceedings of the National Academy of Sciences of the United States of America 110, 7038-7043 (2013).

27. Z. Guo et al., In vivo direct reprogramming of reactive glial cells into functional neurons after brain injury and in an Alzheimer's disease model. Cell Stem Cell 14, 188-202 (2014).

28. P. Rivetti di Val Cervo et al., Induction of functional dopamine neurons from human astrocytes in vitro and mouse astrocytes in a Parkinson's disease model. Nat Biotechnol 35, 444-452 (2017). 


\section{Acknowledgments:}

We would like to thank J.L. Wang, J.Y. Guan, Lin Cheng, M.L. Qu, Xu Zhang, Ting Zhao and other laboratory members for suggestions. This work was supported by the National Key Research and Development Program of China (2016YFA0100100 and 2017YFA0103000), the National Natural Science Foundation of China (31730059 and 31521004), the Guangdong Innovative and Entrepreneurial Research Team Program (2014ZT05S216), the Science and Technology Planning Project of Guangdong Province, China (2014B020226001), the Science and Technology Program of Guangzhou, China (2016B030232001), the Science and Technology Program of Guangzhou, China (201508020001). This work was supported in part by a grant from the BeiHao Stem Cell and Regenerative Medicine Translational Research Institute. 


\section{Figure 1. Lineage tracing system to track the astrocytic origin of converted CiNs.}

(A) Scheme of mGfap-Cre/Rosa26-tdTomato lineage-tracing constructs.

(B) Cell identity characterization of tdTOMATO ${ }^{+}$cells in lineage-tracing mice $(n=3)$.

(C) Immunohistochemistry characterization of tdTOMATO+ cells in lineage-tracing mice. tdTOMATO+ cells were immunopositive for astroglial markers GFAP and S100B. These cells were negative for neuronal markers (NEUN, DCX, NEUROD1 and NEUROG2), microglial marker (IBA1) and oligodendrocyte marker (MBP). Arrowheads point to examples of tdTOMATO labeled cells.

Scale bar: $25 \mu \mathrm{m}$.

Error bars represent s. e. m.

Figure 2. In vivo chemical induction of resident astrocytes into CiNs in adult mouse striatum.

(A) Scheme of the chemical infusion into the mouse brain. (IHC, immunohistochemistry; Ctx, cortex; Cc, corpus callosum; Str, striatum)

(B) Diagram of the distribution of converted neurons in the striatum. Immunohistochemistry analyses of $\mathrm{NEUN}^{+} / \mathrm{tdTOMATO}^{+}$cells in the striatum of chemically treated mice at 8 wpi. High-magnification panels represent the co-localization of NEUN and DAPI. Yellow dots represent $\mathrm{NEUN}^{+} /$tdTOMATO ${ }^{+}$cells in the striatum.

(C) Immunohistochemistry analyses of CiNs at different time points; in tdTOMATO ${ }^{+}$cells, the DCX were detected at 2 wpi; TUJ1, NF-H and GAD67 were detected at 8 wpi. NEUN were detected at both 8 wpi and one year-post-injection. Arrowheads point to examples of CiNs.

(D) Quantifications of $\mathrm{NEUN}^{+} / \mathrm{tdTOMATO}^{+}$cells around the injection site in the striatum of chemically treated mice at 2,5 , and 8 wpi $(n=2)$. 
(E) Quantification of $\mathrm{NEUN}^{+} / \mathrm{tdTOMATO}^{+}$cells at different distances from the injection site at 8 wpi. (intact group, $\mathrm{n}=3$; vehicle group, $\mathrm{n}=5$ and FICBY group, $\mathrm{n}=5$ ).

Scale bars: (B) $75 \mu \mathrm{m}, 20 \mu \mathrm{m}$ in high-magnification panels; (C) $25 \mu \mathrm{m}$.

Error bars represent s. e. m. ${ }^{* * *} P<0.0001$ by one-way ANOVA with Tukey's Multiple Comparison Test.

\section{Figure 3. Electrophysiological analyses of in vivo reprogrammed CiNs.}

(A) Representative images of neuron-like tdTOMATO ${ }^{+}$cells in brain slices for patch-clamp recordings.

(B) Representative single action potential (AP) traces of the CiNs at 4 wpi and 8 wpi.

(C) Whole cell patch-clamp recordings of astrocytes, and the CiNs at 6 wpi and 8 wpi.

(D) Quantification of CiNs firing none, one or more than one spike at various time points during in vivo chemical induction.

(E) Gradual maturation of membrane-intrinsic properties of CiNs from 4 to 12 wpi as shown for membrane capacitance $(\mathrm{Cm})$, input resistance and AP threshold.

(F) Recording of inward currents in CiNs in voltage-clamp mode. Tetrodotoxin (TTX) (500 nM) was used to block voltage-dependent $\mathrm{Na}^{+}$channels $(\mathrm{n}=3)$.

(G) Recording of spontaneous postsynaptic potentials in CiNs. CNQX $(20 \mu \mathrm{M})$ and AP5 $(50 \mu \mathrm{M})$ were used to block spontaneous postsynaptic potentials $(n=4)$.

Scale bar: $20 \mu \mathrm{m}$.

Error bars represent s. e. m. 


\section{Figure 4. Integration of in vivo reprogrammed CiNs into the mouse brain.}

(A) Scheme of the PRV-based retrograde transsynaptic tracing process.

(B) Confocal images of initiating astrocytes (green), CiNs (red and green) and traced host neurons (red) in the chemical induction group and vehicle group $(\mathrm{n}=3)$. Arrowheads point to examples of CiNs. High-magnification panels represent the co-localization of EGFP and DAPI.

(C) Histogram representing the total number of DsRed $^{+} / \mathrm{EGFP}^{+}$cells (CiNs) in the chemical induction group and vehicle group $(n=4)$.

(D) Quantification of DsRed single-positive cells through the injection region $(\mathrm{n}=2)$.

(E) Representative confocal image of an $\mathrm{EGFP}^{+} / \mathrm{DsRed}^{+} \mathrm{CiN}$ and its connecting DsRed singlepositive neuron. White arrowhead points to the $\mathrm{CiN}$, and the asterisk highlight the local neuron which has direct synaptic connections with the CiN.

(F) The three-dimensional reconstruction of (E upper). The arrowhead and asterisk point to the same cells in (E upper).

Scale bars: (B), $100 \mu \mathrm{m}, 20 \mu \mathrm{m}$ in high-magnification panels; (E), $40 \mu \mathrm{m}$.

Error bars represent s. e. m. 


\section{Figure 1}

bioRxiv preprint doi: https://doi.org/10.1101/305185; this version posted April 20, 2018. The copyright holder for this preprint (which was not

A

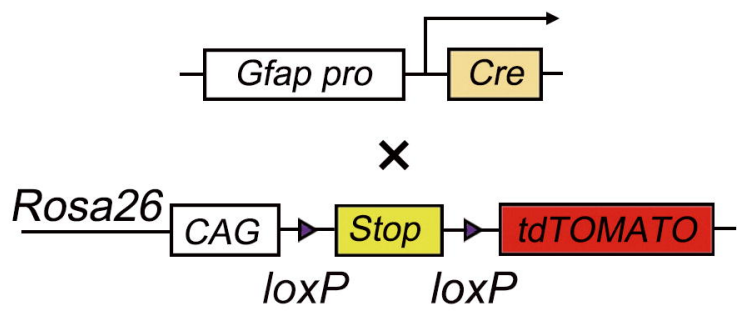

C

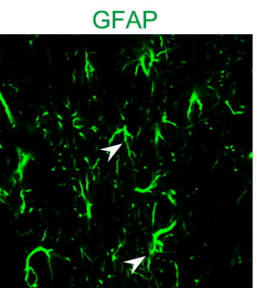

NEUN

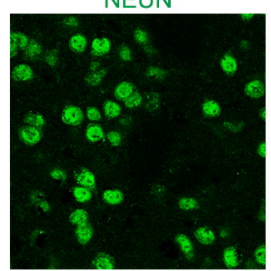

NEUROG2

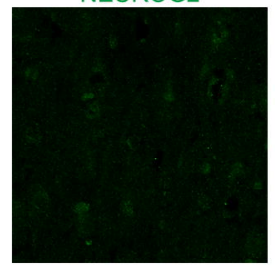

MBP

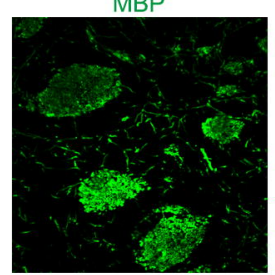

GFAP/tdTOMATO

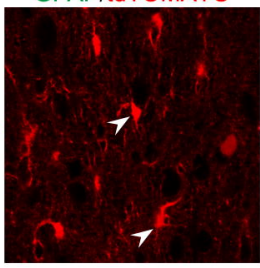

tdTOMATO

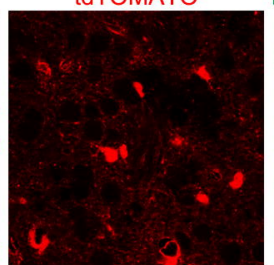

tdTOMATO

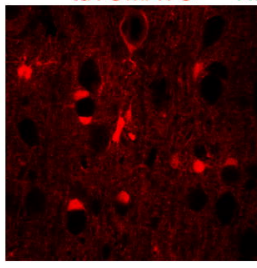

tdTOMATO

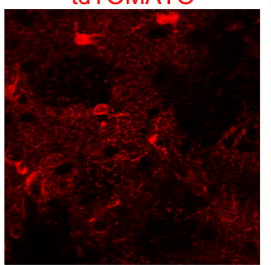

B
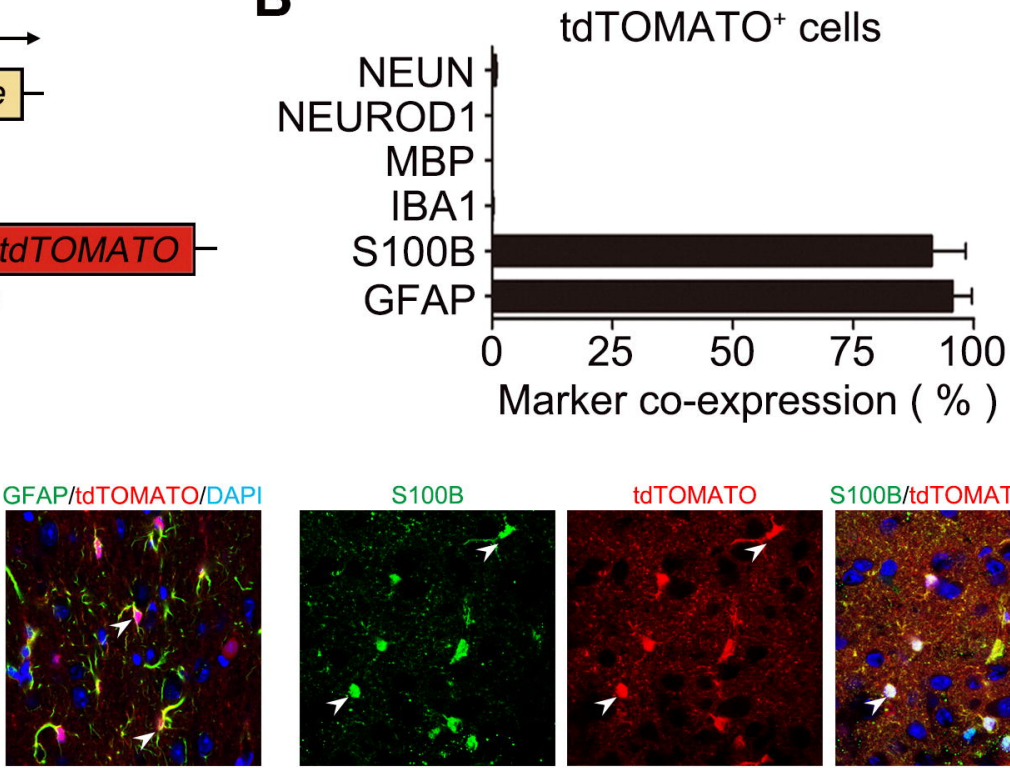

NEUN/tdTOMATO/DAPI
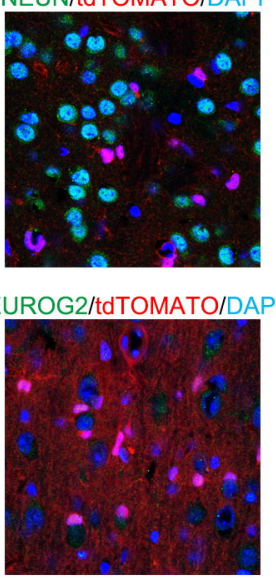

MBP/tdTOMATO/DAPI

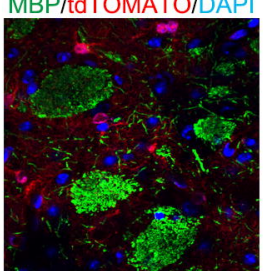

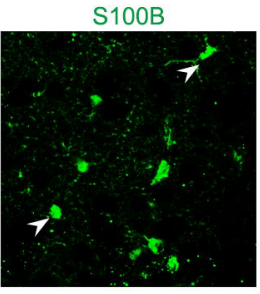

NEUROD1

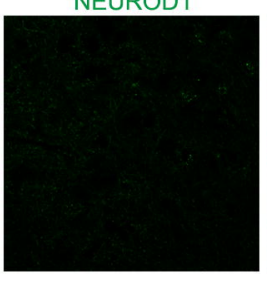

DCX

tdTOMATO

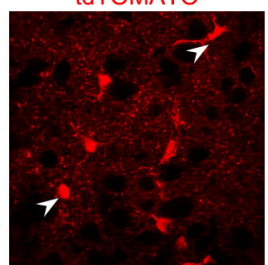

tdTOMATO

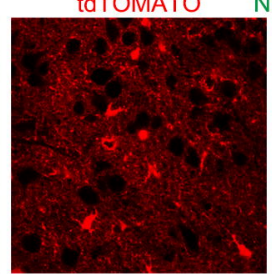

tdTOMATO

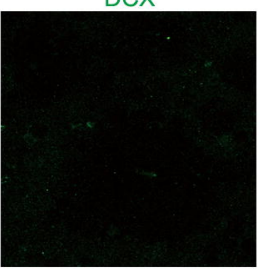

IBA1
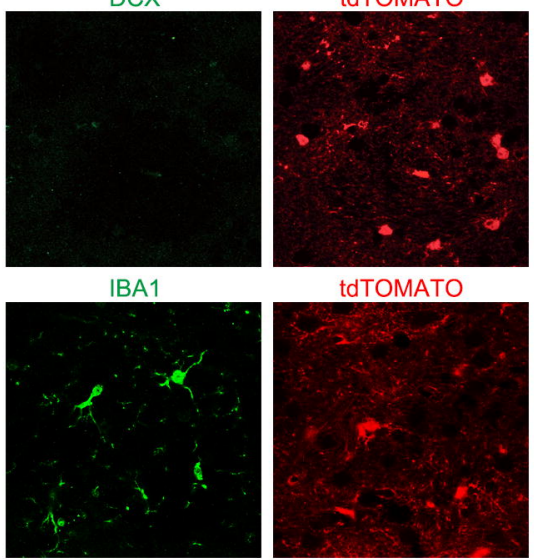

tdTOMATO

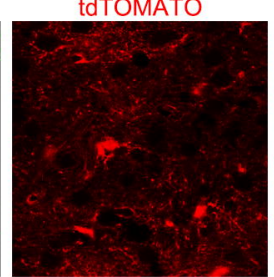

S100B/tdTOMATO/DAPI

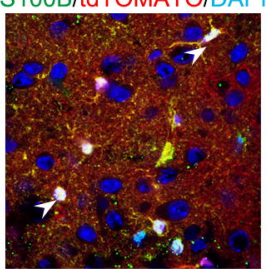

NEUROD1/tdTOMATO/DAP

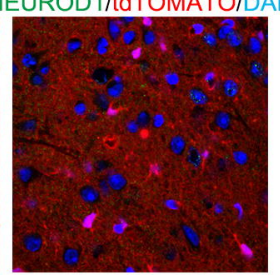

DCX/tdTOMATO/DAPI

IBA1/tdTOMATO/DAPI
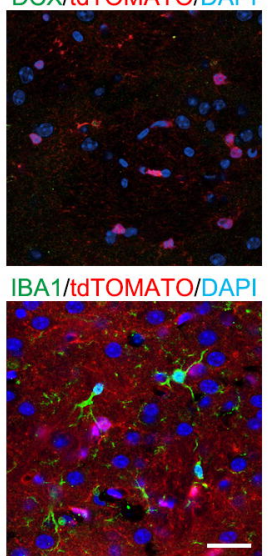
Figure 2

A

B

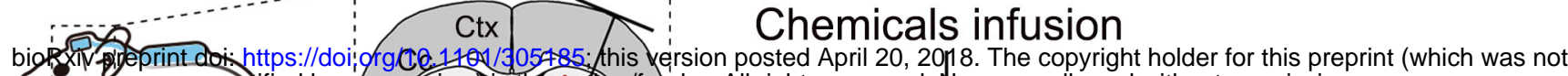

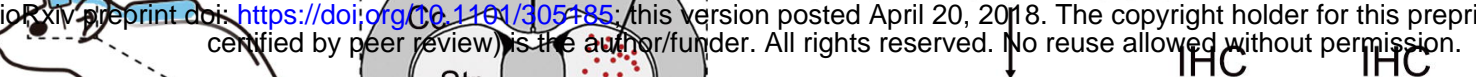
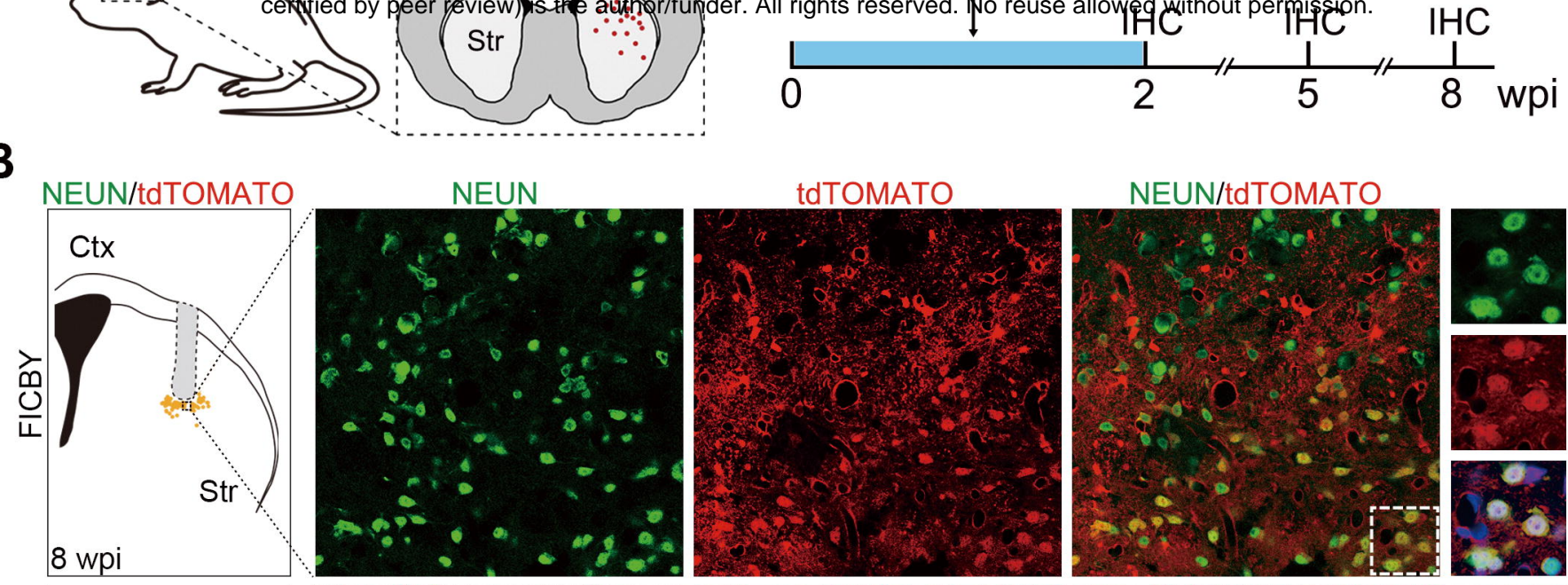

tdTOMATO

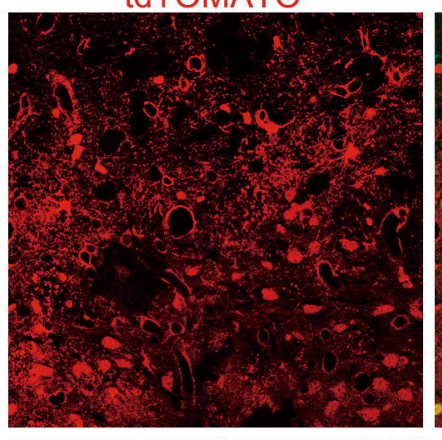

NEUN/tdTOMATO
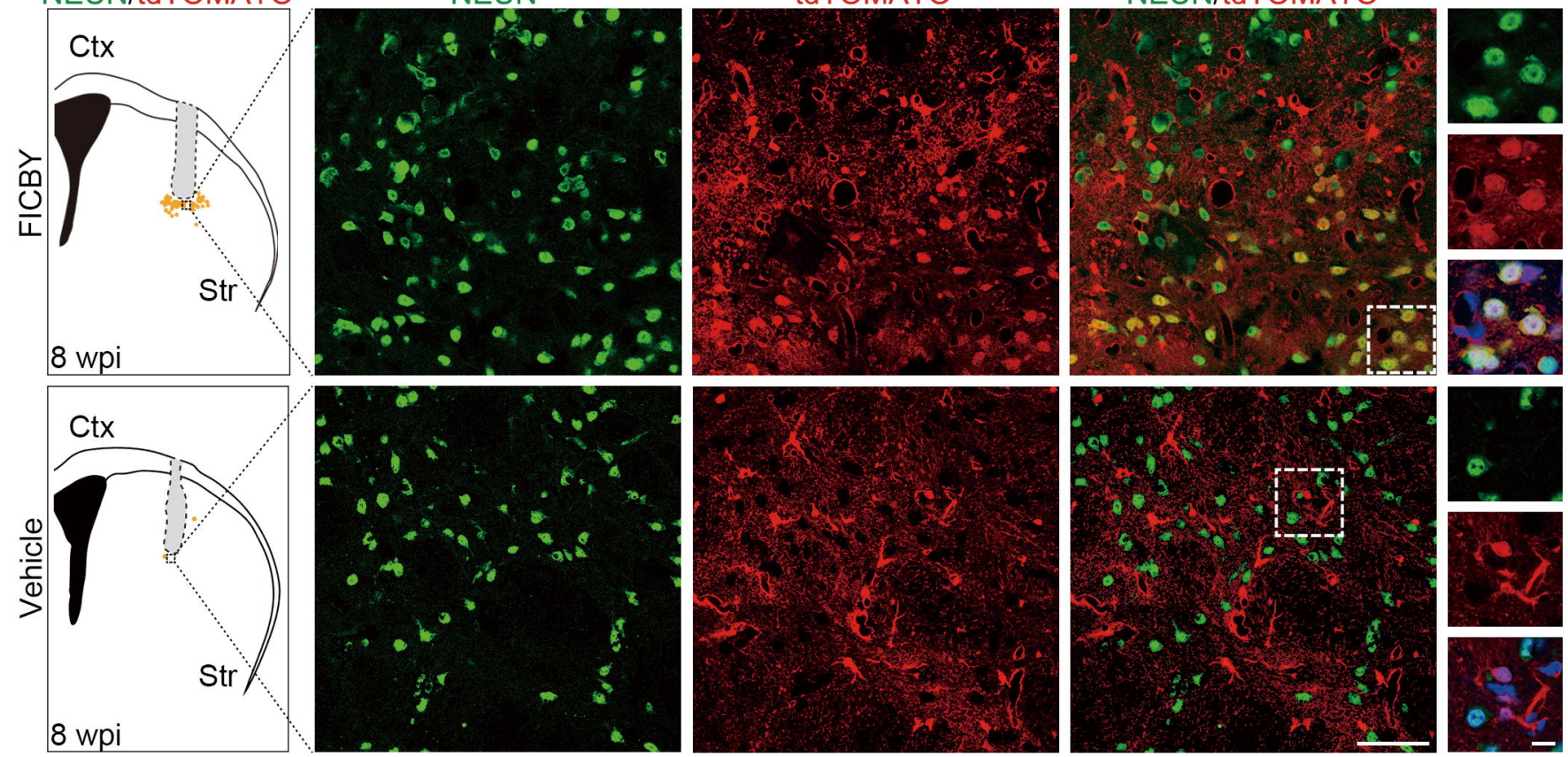
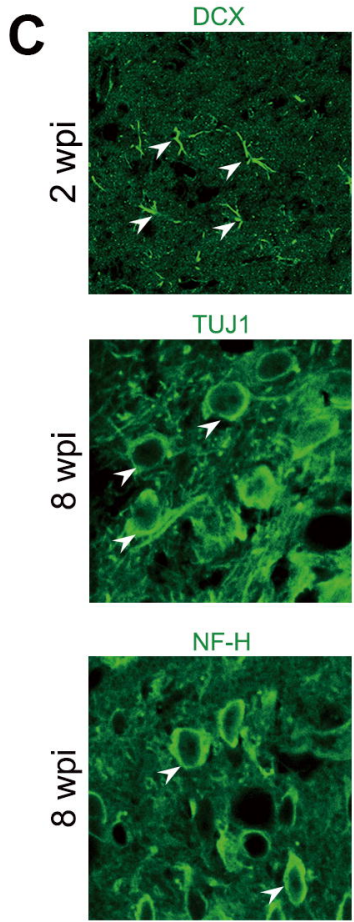

D

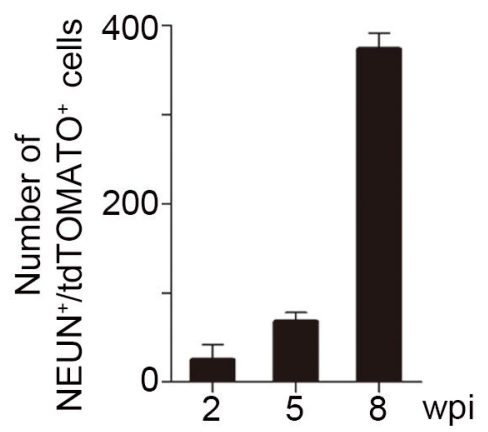

DCX/tdTOMATO

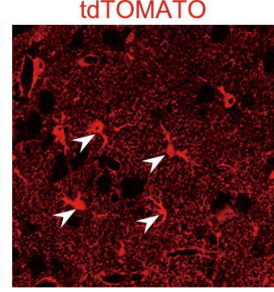

tdTOMATO

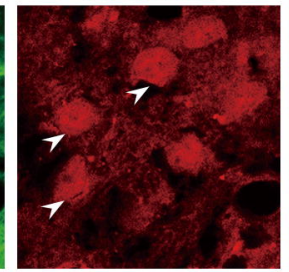

tdTOMATO

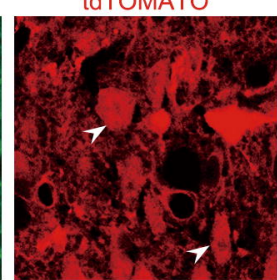

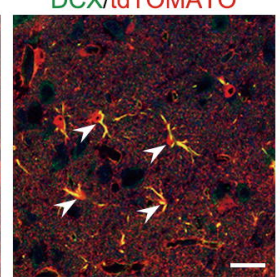

TUJ1/tdTOMATO/DAPI

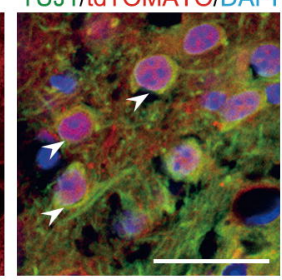

NF-H/tdTOMATO/DAPI

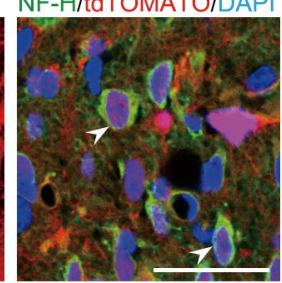

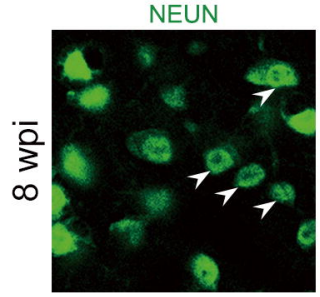
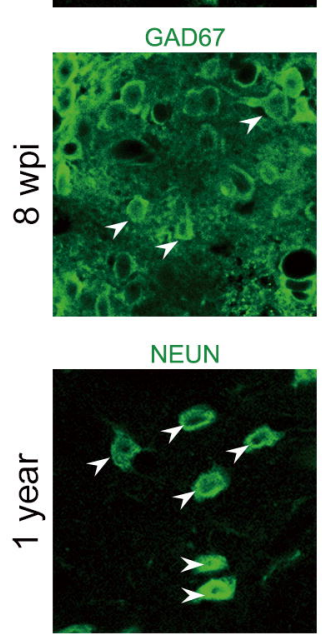

tdTOMATO

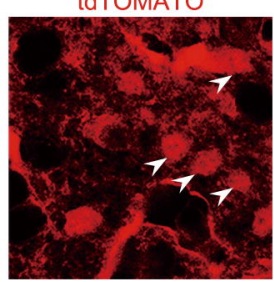

tdTOMATO

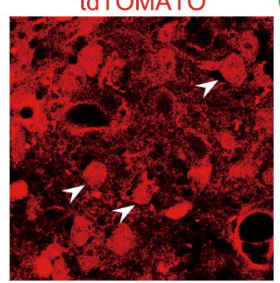

tdTOMATO

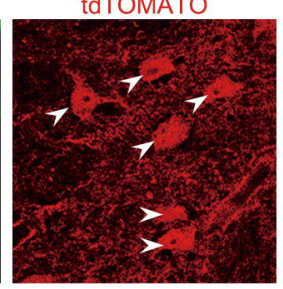

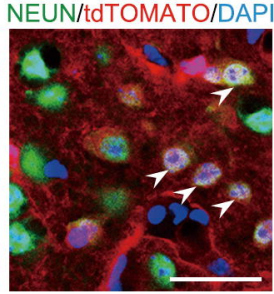

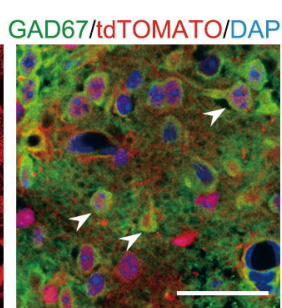

NEUN/tdTOMATO/DAP|

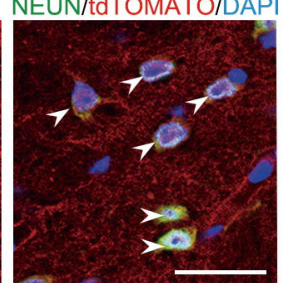

E

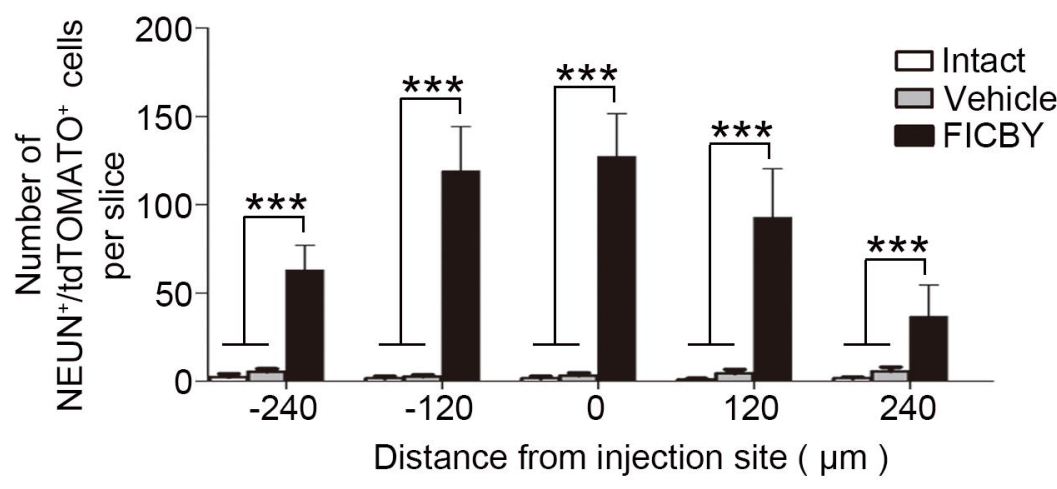


Figure 3

A

bioRxiv preprint doi: https://doi.org/10.1101/305185; this version posted April 20, 2018. The copyright holder for this preprint (which was not
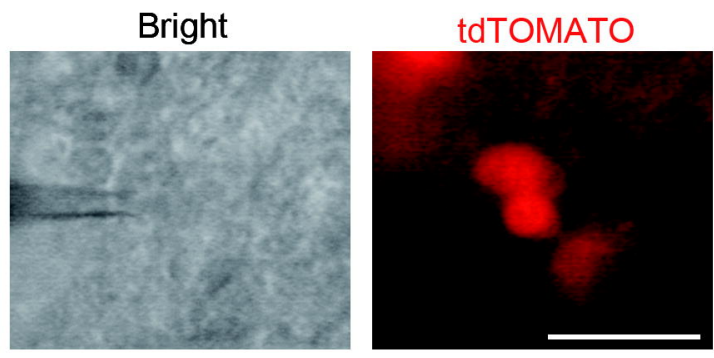

C

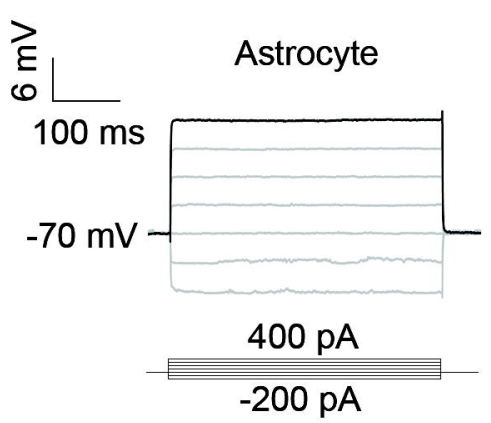

D

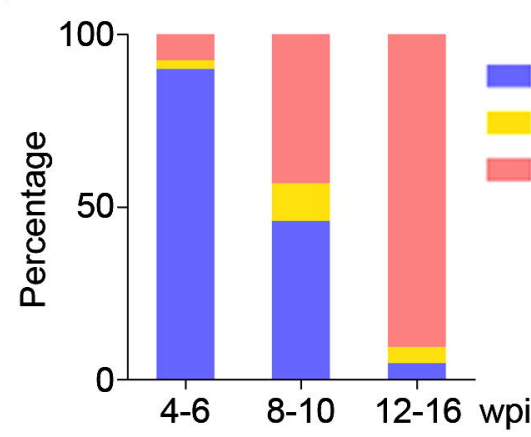

$\mathbf{F}$

$\mathrm{Na}^{+}$currents

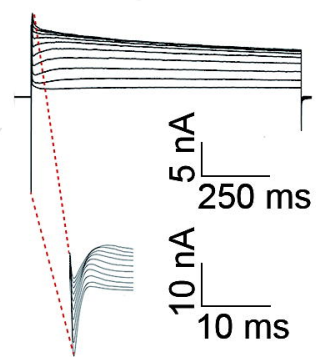

with TTX

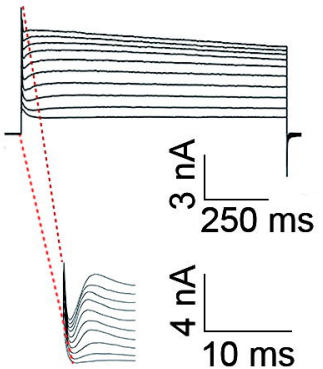

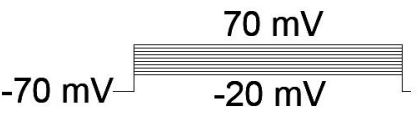

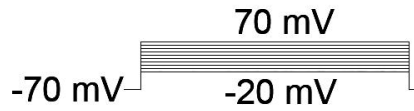

E

no spike

1 spike

$\geq 2$ spikes

능 40
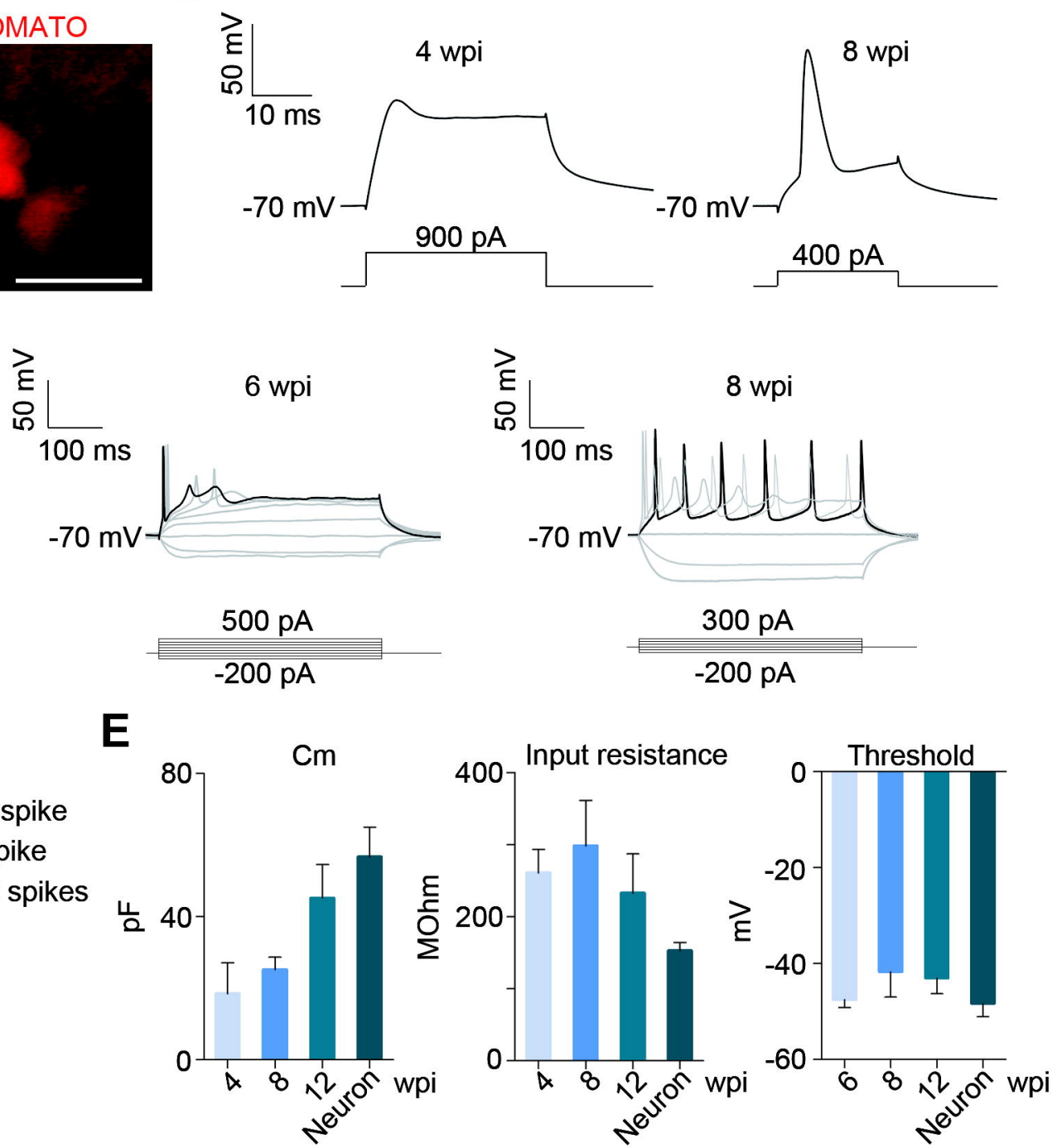

G

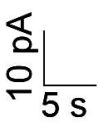

with AP5 and CNQX

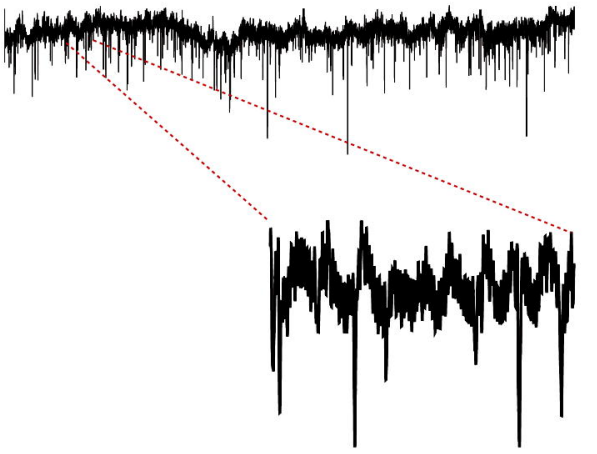

$\ulcorner 5 \mathrm{~s}$

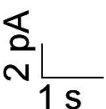




\section{Figure 4}

A

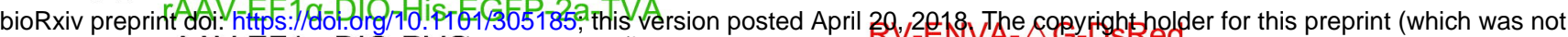

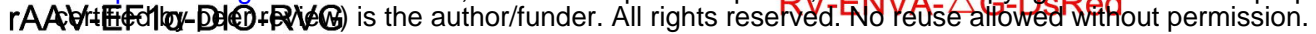

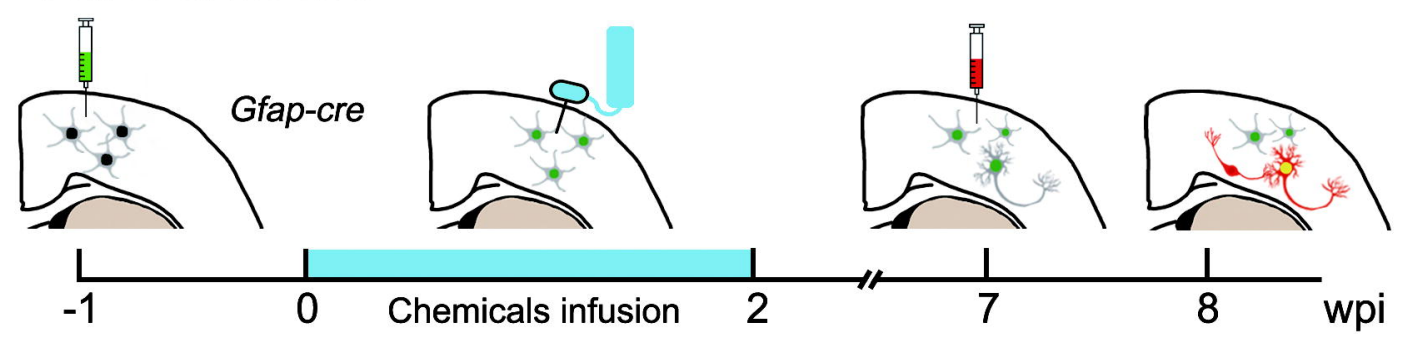

B

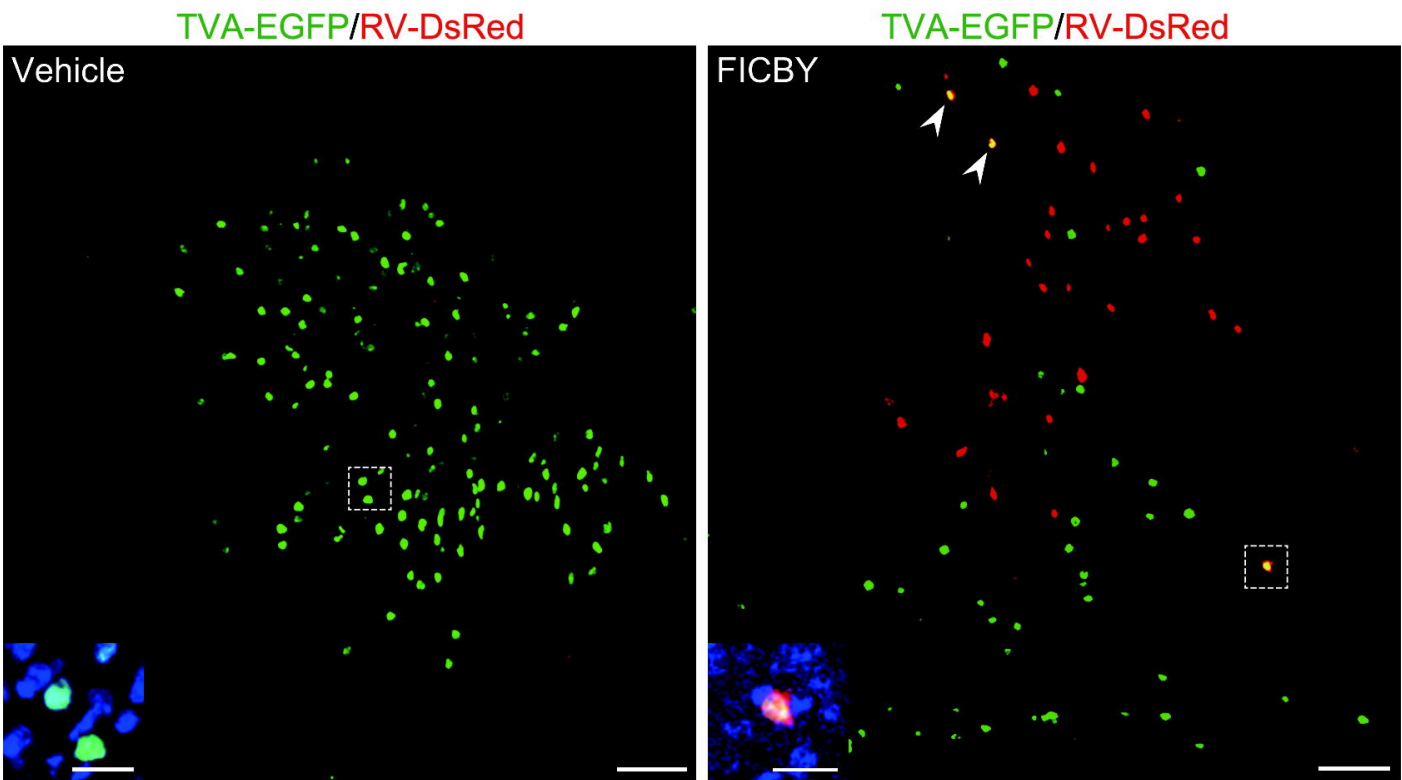

C

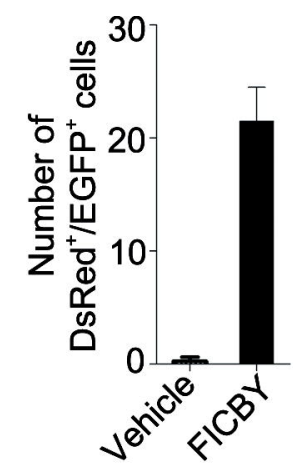

E

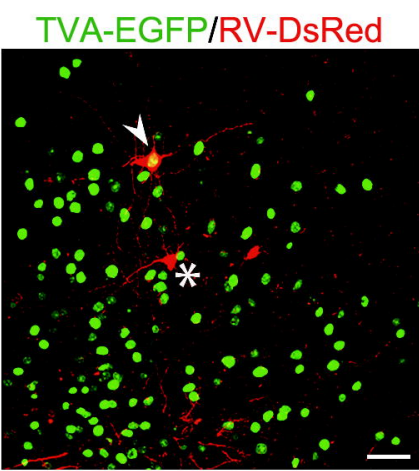

D

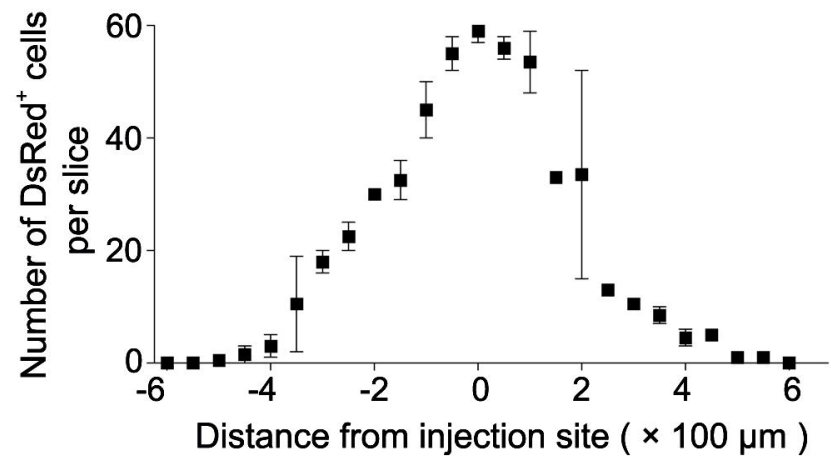

F

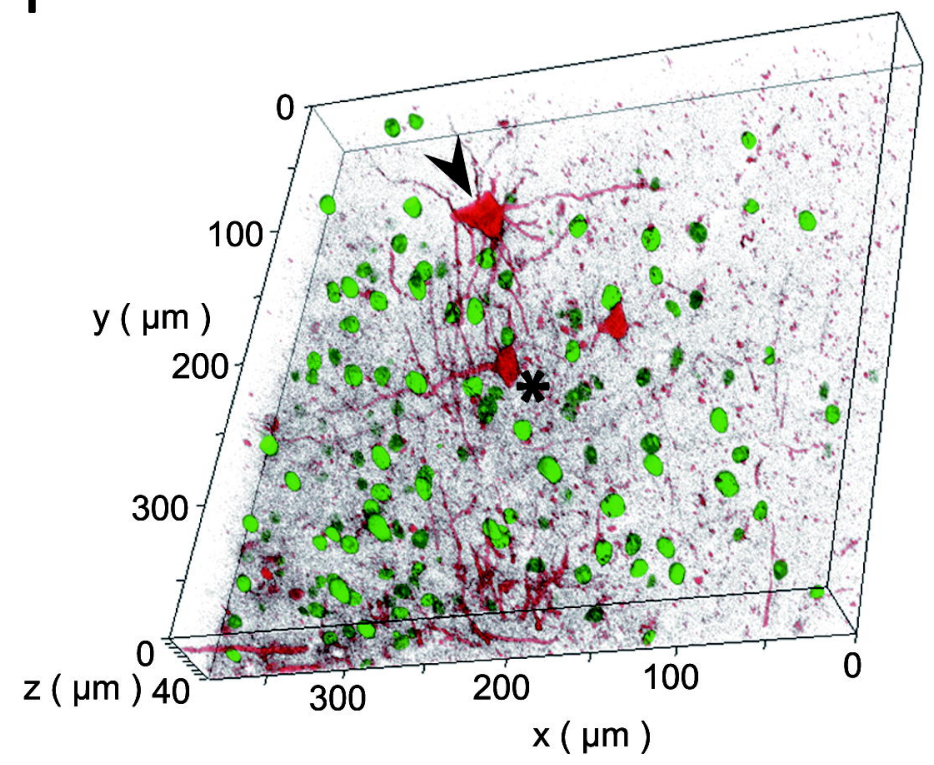

